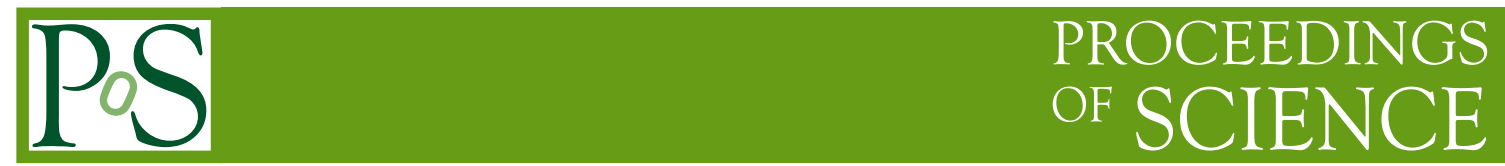

\title{
Lepton Number Violation and Neutrino Masses
}

\author{
Bjoern Lehnert ${ }^{a * *}$ \\ ${ }^{a}$ Nuclear Science Division, Lawrence Berkeley National Laboratory, \\ Berkeley, CA 94720, U.S.A. \\ E-mail: bjoernlehnert@lbl.gov
}

Non-zero neutrino masses are experimentally established since the Nobel-Prize winning discovery of neutrino oscillation. This opens the possibility to distinguish the Majorana and Dirac nature of neutrinos and in turn, allows to search for lepton number violation in the neutrino sector. A lepton number violation through Majorana neutrinos is a prime candidate for creating the baryon number asymmetry and could explain why there is more matter than anti-matter in the Universe. The absolute neutrino mass scale is strongly constrained but still unknown today. Precision $\beta$-decay experiments, cosmological probes and, neutrinoless double beta decay determine the neutrino mass scale with different observables.

This proceedings summarizes the current status of the international experimental efforts aiming to measure the absolute neutrino mass scale and to search for lepton number violation in the neutrino sector.

40th International Conference on High Energy physics - ICHEP2020

July 28 - August 6, 2020

Prague, Czech Republic (virtual meeting)

\footnotetext{
${ }^{*}$ Speaker
} 


\section{Introduction}

Neutrinos are weakly interacting particles which are elusive and difficult to detect experimentally. When they were postulated by Wolfgang Pauli in 1930 (almost exactly 90 yr before ICHEP2020), he feared they were so elusive that they could never be detected. Indeed, it took a quarter of a century until 1956 when the first (anti-)neutrinos were observed in a nuclear reactor experiment by Cowan and Reines [1,2]. Almost from the very beginning, these neutral particles were believed to be extremely light or even massless. They were hypothesized to be their own anti-particles by Ettore Majorana [3] i.e. of Majorana nature as opposed to Dirac nature. It took until 1998 (almost half a century after their discovery), to demonstrate that they are not massless and indeed have non-zero mass eigenstates. The discovery of neutrino oscillation by the SNO, Super-Kamiokande and KamLAND experiments also established that mass eigenstates and flavor eigenstates mix. However, current oscillation experiments only determine mass differences. The absolute neutrino mass scale remains unknown today - albeit strongly constrained. The Majorana or Dirac nature of neutrinos is unknown but investigated by a diverse experimental program of neutrinoless double beta decay experiments.

The PMNS (Pontecorvo-Maki-Nakagawa-Sakata) neutrino mixing matrix connects the lepton flavor and mass eigenstates

$$
\begin{gathered}
\left|\nu_{\text {flavor }}>=\sum_{i} U_{\alpha i}^{*} \cdot\right| \nu_{\text {mass }}> \\
U_{\alpha i}=\left(\begin{array}{ccc}
1 & 0 & 0 \\
0 & c \Theta_{23} & s \Theta_{23} \\
0 & -s \Theta_{23} & c \Theta_{23}
\end{array}\right)\left(\begin{array}{ccc}
c \Theta_{13} & 0 & s \Theta_{13} \cdot e^{-i \delta} \\
0 & 1 & 0 \\
-s \Theta_{13} \cdot e^{-i \delta} & 0 & c \Theta_{13}
\end{array}\right)\left(\begin{array}{ccc}
c \Theta_{12} & s \Theta_{12} & 0 \\
-s \Theta_{12} & c \Theta_{12} & 0 \\
0 & 0 & 1
\end{array}\right)\left(\begin{array}{ccc}
1 & 0 & 0 \\
0 & e^{-i \alpha / 2} & 0 \\
0 & 0 & e^{-i \beta / 2}
\end{array}\right)
\end{gathered}
$$

and contains the three mixing angles $\Theta_{12}, \Theta_{13}$, and $\Theta_{23}$ as well as a complex phase $\delta$. In case of the Majorana nature, neutrino and anti-neutrino states are identical freeing two degrees of freedom which manifest in the two complex Majorana phases $\alpha$ and $\beta$. The mass difference of neutrino eigenstates is shown in the illustration below.

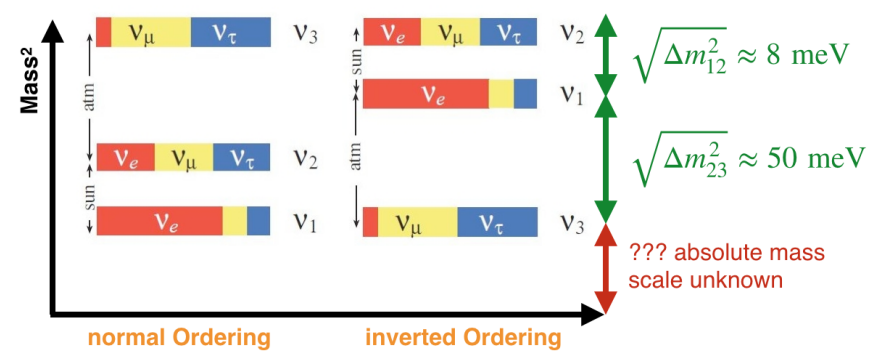

Precise experimental information on $\Delta m_{12}^{2}$ and $\Delta m_{23}^{2}$ comes from oscillation experiments. However, two scenarios remain possible: the normal and inverted ordering or $\operatorname{sign}\left(\Delta \mathrm{m}_{23}^{2}\right)$. The difference is if the electron neutrino flavor predominantly mixes into the lightest mass eigenstate or into a heavier one. Also unknown is the absolute mass scale of the system which can also be expressed as the absolute value of the lightest mass eigenstate $v_{1}$ or $v_{3}$, respectively.

Today, the mixing angles $\Theta_{12}, \Theta_{13}$, and $\Theta_{23}$ and mass splittings $\Delta m_{12}^{2}$ and $\Delta m_{23}^{2}$ are well determined by a variety of precision oscillation measurements (shown in green). The CP phases 
and mass ordering cannot easily be determined in oscillation experiments (shown in yellow) and require subdominant difference in matter interactions between neutrinos and anti-neutrinos of different flavors. Future oscillation experiments will determine these properties within the next 10 years. The determination of the absolute neutrino mass scale and the Majorana nature of neutrinos (shown in red) is challenging and investigated by different experiments. This is the focus of this proceedings.

\section{Neutrino Mass Observables}

Since neutrino mass and flavor eigenstates mix, there is no unique neutrino mass definition and three different observable are investigated. $\beta$-decay experiments measure the effective mass of the electron neutrino which is the incoherent sum of the mass eigenstates mixing into the electron flavor:

$$
m_{\beta}=\sqrt{\sum_{i} m_{i}^{2}\left|U_{e i}\right|^{2}}
$$

This approach is model independent and only relies on the kinematics of the final-state particles in $\beta$-decay.

Cosmological probes measure the total sum of the masses and how this sum influences the evolution of the Universe:

$$
m_{\Sigma}=\sum_{i} m_{i}
$$

This approach depends on the cosmological model and typically assumes $\Lambda \mathrm{CDM}$.

Neutrinoless double beta $(0 v \beta \beta)$ decay experiments measure the effective mass of a virtual electron neutrino propagator and the coherent sum of its mass eigenstates:

$$
m_{\beta \beta}=\left|\sum_{i} m_{i} U_{e i}^{2}\right|
$$

This approach requires lepton number violation to exist and the decay to be dominated by the exchange of a light Majorana neutrino propagator. It is also subject to non-negligible uncertainties in nuclear structure calculations for the nuclear matrix elements. Out of these three observables, only $m_{\beta}$ is model independent.

\section{Neutrino mass from kinematic measurements}

Kinematically, the neutrino mass is easiest observed in $\beta$-decay where a massive neutrino slightly distorts the energy spectrum of the electron. The observed energy distribution in $\beta$-decays was the original motivation for Pauli to postulate the neutrino in the first place, making it a threebody decay. Experimentally, the challenges are to determine a small spectral distortion close to the Q-value (available decay energy) where the count rate drops significantly. A low background, an excellent energy resolution, and a suitable isotope for $\beta$-decay are required. The isotopes being used are tritium ${ }^{3} \mathrm{H}\left(Q_{\beta}=18.6 \mathrm{keV}, T_{1 / 2}=12 \mathrm{yr}\right)$ and ${ }^{163} \mathrm{Ho}\left(Q_{\beta}=2.8 \mathrm{keV}, T_{1 / 2}=4600 \mathrm{yr}\right)$. 

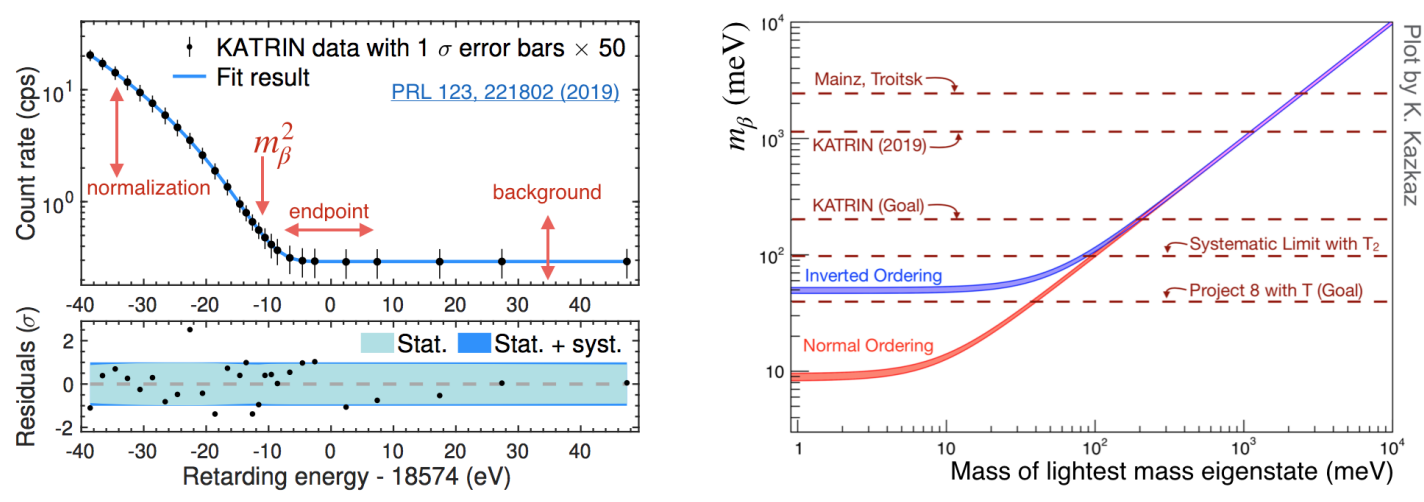

Figure 1: Left: Measured KATRIN energy spectrum and fit of first dataset. The data is well described with four paramters: normalization, endpoint, background and $m_{\beta}^{2}$. Adopted from [5]. Right: $\beta$-decay neutrino mass $m_{\beta}$ vs the lightest mass eigenstate. Allowed parameter space from oscillation experiments is shown in red (NO) and blue (IO). Current and future sensitivity limits are shown. Adopted from [8].

Other kinematic investigations yielded less stringent results on the neutrino mass limits. This include supernova neutrino time-of-flight measurements $\left(m_{\beta}<5.8 \mathrm{eV}\right), \pi$-decay $\left(m_{v_{\mu}}<190 \mathrm{keV}\right)$, and $\tau$-decay $\left(m_{v_{\tau}}<18.2 \mathrm{MeV}\right)$ [4]. The best limit was recently set by the KATRIN experiment at $m_{\beta}<1.1 \mathrm{eV}[5]$.

The KATRIN (Karlsruhe Tritium Neutrino) experiment uses a windowless gaseous molecular tritium $\left({ }^{3} \mathrm{H}_{2}\right)$ source and a high precision spectroscopic filter. $\beta$-decay electrons from radioactive decays in the source are transported adiabatically through the spectrometer and only those with sufficient energy are counted in a pixelated Si-detector. The acquired energy spectrum is the integral count rate above a set retarding potential which is scanned through values around the Q-value at $18.6 \mathrm{keV}$. The electrons are emitted isotropically and the main experimental challenge is to probe all electrons equally within a $50 \mathrm{deg}$ emission angle to maximize the signal strength. This is achieved with a MAC-E (Magnetic Adiabatic Collimation and Electrostatic) filter which magnetically aligns the transverse momentum of selected electrons into a longitudinal momentum at the point of highest filter potential. This concept achieves a filter resolution of $\approx 1 \mathrm{eV}$ at $18.6 \mathrm{keV}$ electron energy with a 23-m long and 10-m wide barrel-shaped spectrometer at the end of a 70-m long beam line. These large size requirements will not allow to scale this approach further for better mass sensitivity, without significant changes to the measurement concept e.g. using time-of-flight information to obtain a differential spectrum.

KATRIN started operations in 2019 and is currently the leading neutrino mass measurement using $\beta$-decay. With the first $33 \mathrm{~d}$ of measurement data, existing constraints on $m_{\beta}$ were improved by a factor of two compared to previous limits by the Mainz and Troitzk experiments. The recorded energy spectrum and fit are shown in Fig. 1 (left). The spectrum is well described by four parameters: the normalization, the endpoint energy ${ }^{1}$, a constant background, and the parameter of interest $m_{\beta}^{2}$. The best fit result is $m_{\beta}^{2}=-1.0_{-1.1}^{+0.9} \mathrm{eV}^{2}$, allowing negative values in the Frequentist regime. The probability for this result in $(-\infty,-1.0] \mathrm{eV}^{2}$ is $19 \%$. Limits on $m_{\beta}$ are obtained with the Lokhov-

${ }^{1}$ The endpoint is a free parameter since small potential offsets in the setup decouple it from the well known Q-value of the decay within the required precision. 
Tkachov method [6] $(<1.1 \mathrm{eV})$, the Feldman-Cousins method [7] $(<0.8 \mathrm{eV})$, and a Bayesian analysis with flat positive prior in $m_{\beta}^{2}(<0.9 \mathrm{eV})$. The uncertainties are dominated by statistics at $\pm 0.97 \mathrm{eV}^{2}$. A number of systematic effects are included $\left( \pm 0.32 \mathrm{eV}^{2}\right)$ of which background uncertainties are the dominant source. The collaboration will acquired $3 \mathrm{yr}$ of data within $5 \mathrm{yr}$ and aims at a final sensitivity of $0.2 \mathrm{eV}(90 \% \mathrm{CL})$ or a discovery potential of $0.35 \mathrm{eV}(3 \sigma)$.

Two experimental programs are ongoing to develop technology to go beyond the final KATRIN sensitivity. Project 8 [8] employs CRES (Cyclotron Radiation Emission Spectroscopy) to achieve higher energy resolution when measuring ${ }^{3} \mathrm{H} \beta$-decay electrons. This allows a differential energy spectrum to be recorded which contains more information than the integral rate. The collaboration also pursues the use of an atomic ${ }^{3} \mathrm{H}$ source which would eliminate complex final state distributions due to molecular excitations in ${ }^{3} \mathrm{H}_{2}$. The ultimate projected limit of $m_{\beta}$ is at the $\approx 100 \mathrm{meV}$ level. Project 8 has a multi-stage experimental program to develop each technology component. It recently showed the first measured $\beta$-spectrum with ${ }^{3} \mathrm{H}_{2}$ using CRES.

The projected sensitivity for KATRIN and Project 8 is shown in Fig. 1 (right). Shown is $m_{\beta}$ versus the lightest neutrino mass eigenstate with allowed parameter space in red (NO) and blue (IO) from oscillation experiments. With the ultimate sensitivity goal, Project 8 will be able to scrutinize the inverted mass ordering.

Another experimental program explored by the ECHo and HOLMES collaborations uses ${ }^{163} \mathrm{Ho}$, which undergoes electron capture. This probes the neutrino mass as opposed to the anti-neutrino mass in ${ }^{3} \mathrm{H} \beta$-decay. ${ }^{163} \mathrm{Ho}$ has a lower Q-value at $2.83 \mathrm{keV}$, accentuating the neutrino-mass-induced spectral distortion compared to ${ }^{3} \mathrm{H}$ given the same energy resolution. Both experiments use pixelated cryogenic bolometers in which ${ }^{163} \mathrm{Ho}$ is implanted. ECHo is using Metallic Magnetic Calorimeters (MMC) [9] and HOLMES is using Transition Edge Sensors (TES) [10]. This source=detector concept allows a calorimetric measurement of the total decay energy (minus the neutrino energy) regardless of the different final states in the ${ }^{163} \mathrm{Ho} \rightarrow{ }^{163} \mathrm{Dy} *+v_{e} \rightarrow{ }^{163} \mathrm{Dy}+E$ system. Recent advances in the detailed theoretical description of the energy spectrum lead to the sensitivity at $m_{\beta}<150 \mathrm{eV}$ set by ECHo [11]. Both collaborations pursue a staged experimental program and expect a sensitivity $m_{\beta}<20 \mathrm{eV}$ in 2021, $m_{\beta}<2 \mathrm{eV}$ around 2022, and ultimately $m_{\beta}<0.2 \mathrm{eV}$ in the future.

\section{Neutrino mass from cosmology}

Cosmological observations can constrain the total sum of neutrino masses $m_{\Sigma}$ which affect the structure formation in the Universe. Heavy neutrinos wash out gravitational wells smaller than their free-streaming length; thus, heavy neutrinos disfavor smaller structures. The effect is independent of the neutrino flavor and the mass of individual eigenstates. Hence only the total sum of masses is probed. The experimental information comes from observations of the matter distribution e.g. from the power spectrum obtained by galaxy surveys, the anisotropies in the cosmic microwave background (CMB), CMB lensing, etc. However, in order to extract informations on $m_{\Sigma}$ in fits to one or multiple dataset, a cosmological model is assumed making $m_{\Sigma}$ model dependent.

Nevertheless, cosmological bounds give the strongest constraints on neutrino masses to date with current limits at $m_{\Sigma}<120 \mathrm{meV}(95 \% \mathrm{CL})$ from the Planck and BAO data [12]. Fig. 2 illustrates the current limits and future sensitivities. $m_{\Sigma}$ is plotted vs $m_{\beta}$ with allowed regions in 


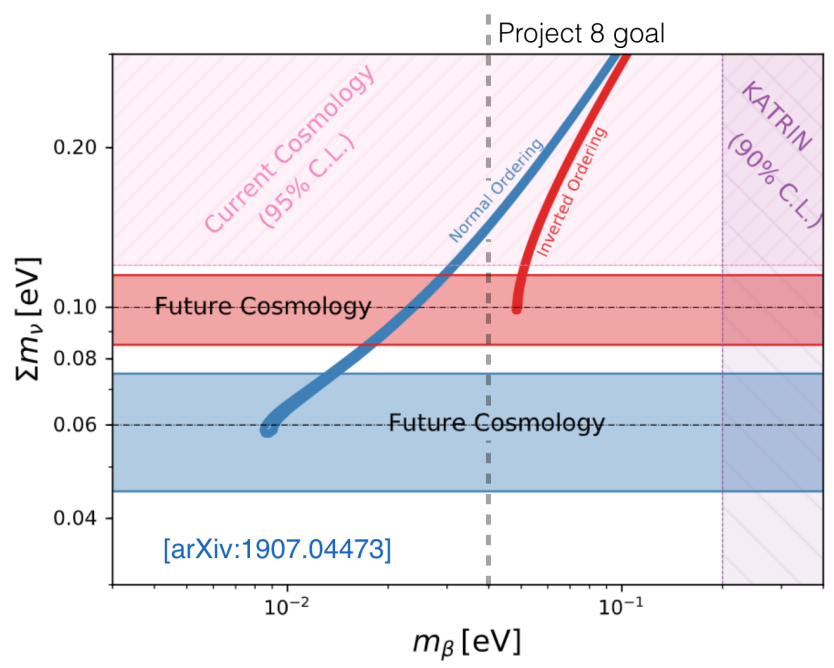

Figure 2: Cosmology neutrino mass observable $m_{\Sigma}$ vs $\beta$-decay neutrino mass observable $m_{\beta}$. The allowed parameter space from oscillation experiments is shown in blue (NO) and red (IO). Future sensitivities from cosmology and $\beta$-decay measurements are shown. Adopted from [13].

blue and red coming from the normal and inverted mass ordering. The $\mathrm{x}$-axis shows $m_{\beta}$ from $\beta$ decay experiments, including future KATRIN and Project 8 sensitivities. The projected sensitivities of cosmological probes aim at $m_{\Sigma}=20 \mathrm{meV}$ [13] using CMB-S4 together with a variety of new ground-based observatories and satellite missions. These probes will differentiate the mass ordering with 2-4 $\sigma$, depending on the mass ordering in nature. It will be particularly interesting when the mass ordering is determined by entirely independent approaches, i.e. by cosmological probes, by neutrino oscillation using matter effects, and by kinematic $\beta$-decay measurements. These results might be available within the next decade. In the worst case, they lead to a convincing cross check of the mass order and the absolute mass scale. In the best case, any tension between the probes will be a hint towards new physics.

A long term dream of neutrino physics is the discovery of the cosmic neutrino background $(\mathrm{C} v \mathrm{~B})$, which is being explored by the PTOLEMY collaboration [14]. Low energy $\mathrm{C} v \mathrm{~B}$ neutrinos at $1.9 \mathrm{~K}$ decoupled before the CMB photons (1 s compared to $379000 \mathrm{yr}$ after the big bang). They are the most abundant matter particles in the Universe, but not energetic enough to be detected directly. However, they can induce $v_{e}$-capture via ${ }^{3} \mathrm{H}+v \rightarrow{ }^{3} \mathrm{He}+\mathrm{e}^{-}$, which results in a discrete peak about two $m_{\beta}$ above the ${ }^{3} \mathrm{H} \beta$-decay endpoint energy. The extremely small interaction rates and narrow energy gap to the endpoint require a vast expansion of the ${ }^{3} \mathrm{H}$ target size, considerable improvements of the energy resolution, and excellent control of systematic uncertainties. There is currently no clear roadmap to achieve the sensitivity required for a discovery under the standard Big Bang model. However, an observation would not only show the existence of $\mathrm{C} v \mathrm{~B}$ neutrinos but also be the ultimate neutrino mass measurement. In addition, the induced $v_{e}$-capture excludes anti-neutrino interaction within the non-relativist $\mathrm{C} v \mathrm{~B}$ distribution, potentially allowing to probe the Majorana-Dirac nature of neutrinos if the local density can be measured and predicted precisely. 


\section{Lepton number violation and neutrino mass from double beta decay}

Double beta decay (DBD) mainly probes lepton number violation (LNV) and can only constrain the neutrino mass $m_{\beta \beta}$ under a specific model. DBDs are second-order weak nuclear decays with can be observed in even-even nuclei whose single $\beta$-decay is strongly disfavored or forbidden. In nature, these configurations only exist in 35 out of about 3000 known isotopes. The decay may occur in two modes:

$$
\begin{array}{ll}
2 v \beta \beta: & (Z, A) \rightarrow(Z+2, A)+2 e^{-}+2 \bar{v}_{e} \\
0 v \beta \beta: & (Z, A) \rightarrow(Z+2, A)+2 e^{-}
\end{array}
$$

where $2 v \beta \beta$ decay is a Standard Model process that has been observed in 11 isotopes. Experimentally, only the electron energy is measured which follows a continuous spectrum similar to $\beta$-decay. In $0 v \beta \beta$ decay, the electrons contain the total decay energy and the experimental signature is a peak search at the Q-value of the decay. This decay mode has not yet been observed and would violate lepton number by two units since no anti-neutrinos are present in the final state. Its discovery would also imply the Majorana nature of neutrinos [15] - albeit only a small Majorana mass component is guaranteed [16].

LNV has vast implications on cosmology and could explain the observed matter-antimatter asymmetry in the Universe. One of the favored ways to create Majorana neutrino mass terms is the see-saw mechanism, which predict heavy Majorana neutrinos at the GUT scale. Those neutrinos could satisfy some of the Sakharov conditions if they live long enough to decay out of equilibrium in the early Universe and decay predominantly into neutrinos as opposed to anti-neutrinos. The generated lepton number (L) could be converted into a baryon number (B) at the electroweak symmetry breaking through theories conserving B-L (see e.g. [17]).

The most favored LNV mechanisms triggering DBD is light Majorana neutrino exchange in which the mass of the virtual neutrino propagator $m_{\beta \beta}$ is connected with the experimentally measured half-life via:

$$
\left(T_{1 / 2}\right)^{-1}=G^{0 v} \cdot\left|M^{0 v}\right|^{2} \cdot\left|m_{\beta \beta}\right|^{2} .
$$

$G^{0 v}$ is the phase space factor and $M^{0 v}$ is the nuclear matrix element (NME) unique for each isotope. $G^{0 v}$ is well known but $M^{0 v}$ has large theoretical uncertainties that stem from nuclear model calculations with their intrinsic difficulties to describe $O(100)$ nucleon systems. Currently, NME's are uncertain by up to a factor 3 which translates in a span of $m_{\beta \beta}$ of about an order of magnitude, given a measured half-life. Reducing these uncertainties is an active field of research [19]. Measurements of $2 v \beta \beta$ decays, its spectral shape [20] or their excited state transitions [21] can help improve nuclear models by adding experimental information for parameter tuning and cross checks.

The observable $m_{\beta \beta}$ is composed of the coherent sum of mass eigenstates which contains the two complex Majorana phases as illustrated in Fig. 3. It is plotted against the lightest mass eigenstates with the allowed parameter regions for the normal and inverted mass ordering scenarios. In the case of normal ordering, the complex phases can cancel the observable for certain combinations. For the inverted ordering, a lower limit exists since the $v_{e}$ flavor is primarily composed of a heavy 


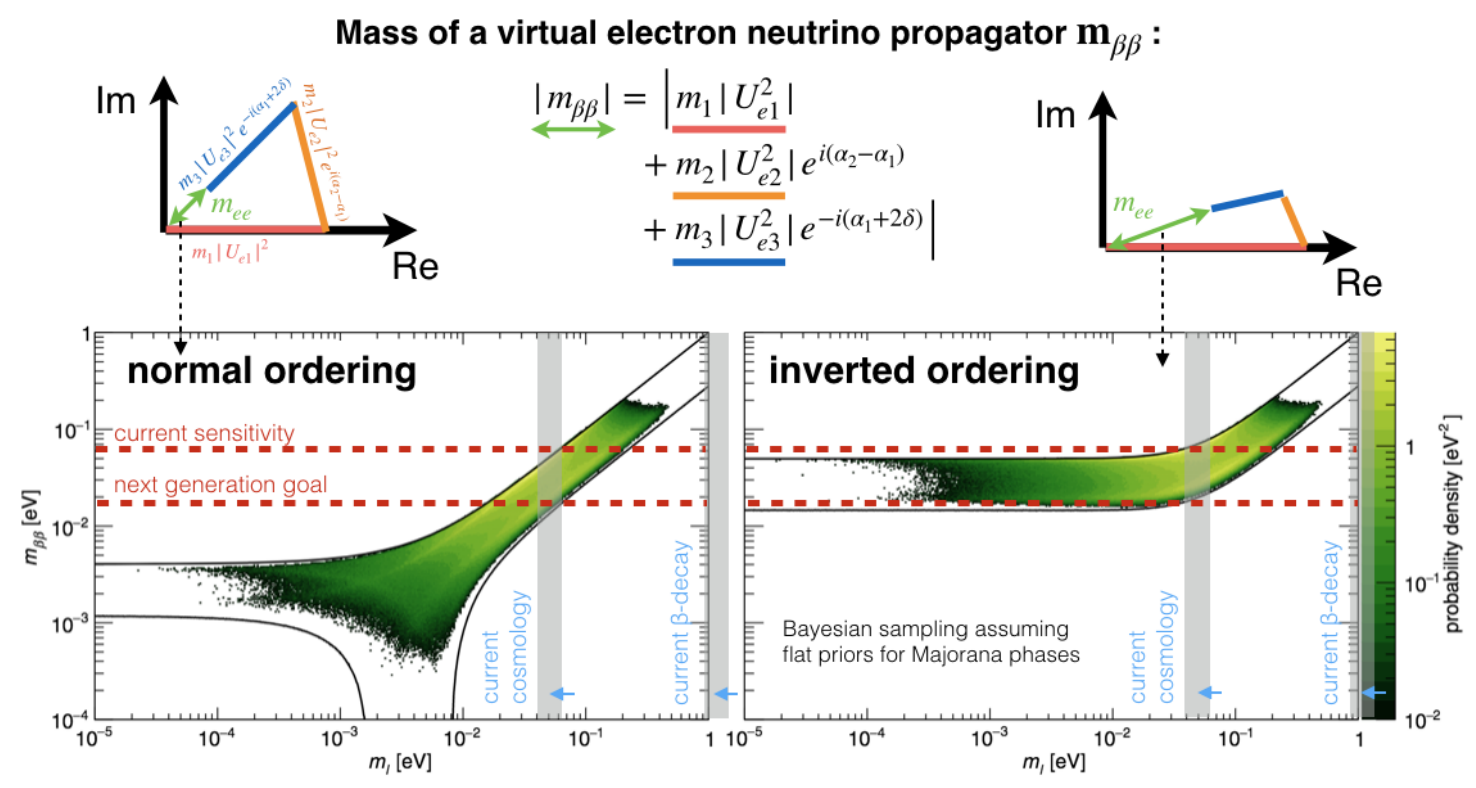

Figure 3: Neutrinoless double beta decay observable for light Majorana exchange $m_{\beta \beta}$. It is composed of the coherent sum of mass eigenstates in a virtual electron neutrino propagator. The complex phases lead to two possible combination: If the dominant mass eigenstate in the electron flavor is small, the other components can cancel the observable (normal ordering). If the the dominant mass eigenstate is heavy, the observable has a lower limit (inverted ordering). The plots show the allowed parameter space for both cases and the color code is a Bayesian probability distribution assuming flat priors for unknown parameters (adopted from [18]). This illustrates the most likely parameter values under this assumption. Also shown are current and future sensitivities from DBD experiments and current limits from cosmology and $\beta$-decay.

mass eigenstate ${ }^{2}$.

A rich experimental program is searching for $0 v \beta \beta$ decay in various isotopes with different detector technologies. All experiments share the common challenge to acquire a large exposure while keeping extremely low background levels. Exposures on the order of $10^{28}$ nuclide $\times$ yr requires isotopically enriched material on the tonne-scale. Background levels on the order of 1 counts per tonne per year in the energy region of interest have to be achieved to discriminate the decay from radioactive background. State-of-the-art DBD experiments have excellent energy resolution for the signal-peak search. They are constructed of ultra-pure materials and use active and passive background mitigation techniques. The experimental program can be broadly separated into 4 main categories: large scintillating detectors, time projection chambers (TPC), cryogenic bolometers and semi-conductor detectors ${ }^{3}$. The following is a brief overview; a more complete description can be found in [18, 22].

Liquid scintillator experiments [23] have the advantage of being easily scalable to large target masses which benefits self-shielding from external background radiation. They are also multipurpose detectors for physics beyond the search for DBD. Their disadvantage is a typically poorer

\footnotetext{
${ }^{2}$ Note that this picture changes if new physics is added, e.g. through a 4th sterile neutrino mass eigenstate.

${ }^{3}$ This is structured similarly to the plenary talks at Neutrino2020 https://conferences.fnal.gov/nu2020/, which was held a month prior to ICHEP2020. Some of the information is not yet published and the recorded talks are referenced for brevity.
} 
energy resolution which makes the identification and reduction of background harder. KamLANDZEN is using ${ }^{\text {enr }} \mathrm{Xe}\left(91 \%{ }^{136} \mathrm{Xe}\right)$ embedded in $1 \mathrm{kT}$ of liquid scintillator in Kamioka, Japan. The previous operation phase, KLZ-400 (400 kg Xe), holds the leading $m_{\beta \beta}$ sensitivity at $<61-165 \mathrm{meV}$ (depending on the NME) with a half-life limit of $>1.1 \cdot 10^{26} \mathrm{yr}$. The collaboration recently doubled

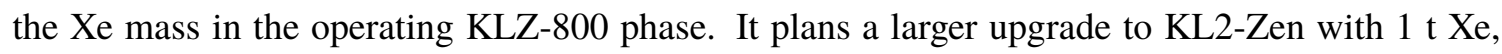
better light collection, new electronics, and a scintillating balloon. SNO+ is a $780 \mathrm{t}$ liquid scintillator detector currently under commissioning in the old SNO facility in SNOLAB, Canada. Here, the $0 v \beta \beta$ decay search is performed with ${ }^{\text {nat }} \mathrm{Te}\left(34 \%{ }^{130} \mathrm{Te}\right)$. The target mass can be easily scaled by increasing the Te loading from $0.5 \%(1.3 \mathrm{t} \mathrm{Te})$ in the beginning to a maximum of $2.5 \%$. At maximum loading, the projected half-life sensitivity is $10^{27} \mathrm{yr}$.

TPCs for DBD searches [24] have the advantage of a large monolithic volume with good self-shielding and particle identification. For liquid and gas Xe TPCs there exist experimental programs to extract and detect the daughter isotope from the Xe target (Barium tagging) which would help to distinguish a DBD event from background processes. Liquid Xe single phase TPCs such as the EXO-200 and nEXO experiments read out charge and scintillation light using an ${ }^{\text {enr Xe }}$ target. EXO-200 at WIPP (Waste Isolation Pilot Plant), USA, has completed. Its half-life limit is $T_{1 / 2}>3.5 \cdot 10^{25} \mathrm{yr}$. $\mathrm{nEXO}$ is a future DBD experiment, which is planned to be constructed at SNOLAB with $5 \mathrm{t}{ }^{\text {enr }} \mathrm{Xe}$ and a sensitivity target of $T_{1 / 2}>10^{28} \mathrm{yr}$ and $m_{\beta \beta}<6-18 \mathrm{meV}$. High pressure gas Xe TPCs, such as the NEXT program at Canfranc, Spain, record electron tracks useful for event classification by topology. A multi-stage program with the completed NEXT-White, the current NEXT-100, and the future NEXT-HD and NEXT-BOLD successively increases the sensitivity to the $10^{28} \mathrm{yr}$ level. Liquid Xe dual phase TPCs in dark matter searches such as Xenon1t, Xenon-nt, LZ, and future DARWIN are also sensitive to ${ }^{136}$ Xe DBD. These dark matter searches use large target masses of natural Xe $\left(9 \%{ }^{136} \mathrm{Xe}\right)$ e.g. $50 \mathrm{t}$ for DARWIN. A recent study suggests a competitive half-life sensitivity of $2.4 \cdot 10^{27} \mathrm{yr}$ in DARWIN. Notably, the first observation of $2 v$ double electron capture ${ }^{4}$ was recently made by Xenon-1t with $T_{1 / 2}=1.8 \cdot 10^{22} \mathrm{yr}$ [25], the longest directly measured half-life of any isotope to date.

Cryogenic bolometers [26] have excellent energy resolution. They are segmented into smaller detectors allowing easy coincidence rejection of background processes. Different crystal types can contain a variety of DBD isotopes making this technology flexible. The disadvantages of segmented detectors are their complexity and expense when scaling to larger target masses. CUROE, currently operated at LNGS (Laboratori Nazionali del Gran Sasso), Italy, uses heat signals in ${ }^{\text {nat }} \mathrm{TeO}_{2}$ crystals which are deployed in the worlds largest $\mathrm{mK}$ cryostat. Its current limits are $T_{1 / 2}>3.2 \cdot 10^{25} \mathrm{yr}$ and $m_{\beta \beta}<75-350 \mathrm{meV}$ with the sensitivity limited by alpha background. The successor collaboration CUPID plans to eliminate this background by particle ID using heat and light signals in $\mathrm{Li}_{2}^{100} \mathrm{MoO}_{4}$ crystals. The CUPID experiment will be operated at the same facility as CUORE. Its sensitivity goals are $T_{1 / 2}>10^{27} \mathrm{yr}$ and $m_{\beta \beta}<10-20 \mathrm{meV}$.

Semi-conductor detectors [27] share similar advantages and disadvantages to cryogenic bolometers. Enriched HPGe detectors $\left(88 \%{ }^{76} \mathrm{Ge}\right)$ are used in the GERDA (LNGS) and MAJORANA (SURF - Sanford Underground Research Facility, USA) experiments. These ${ }^{76} \mathrm{Ge}$ experiments have the lowest background and the best energy resolution in the ROI, as well as the best half-life limit

\footnotetext{
${ }^{4}$ Inverse process to double beta decay $2 v \epsilon \epsilon:(Z, A)+2 e^{-} \rightarrow(Z-2, A)+2 v_{e}$
} 
among DBD searches. The recently published final GERDA results give $T_{1 / 2}>1.8 \cdot 10^{26} \mathrm{yr}$ and $m_{\beta \beta}<80-182 \mathrm{meV}$. Both experiments are combined into the two-stage LEGEND program with LEGEND-200 and LEGEND-1000. The half-life sensitivity goals are $10^{27} \mathrm{yr}$ and $10^{28} \mathrm{yr}$, respectively.

If $0 v \beta \beta$ decay were discovered, it is not immediately clear what mechanism induces this LNV process. Beyond the favored theory of light Majorana neutrino exchange, there are other LNV models, such as Higgs triplets, SYSY particles, or right handed currents, that could trigger the decay [28]. Fig. 3 shows the current and future sensitivities for light Majorana neutrino exchange. However, should another LNV process be dominant, those plots are not applicable. The Majorana nature of neutrinos will be guaranteed by the Schechter-Valle theorem [15]. Additional information can come from observations in multiple isotopes. The more generic version of Eq. 6:

$$
\left(T_{1 / 2}\right)^{-1}=G^{0 v} \cdot\left|\sum_{\text {mech i }} M_{i}^{0 v} \cdot \eta_{i}\right|^{2}
$$

can be constrained through combinations of $M_{i}^{0 v}$ in different isotopes. A worldwide experimental program with different isotopes is important to determine the nature of LNV in the future.

Even more LNV information can be obtained from other signatures in measurements such as di-lepton di-jet events at colliders, rare Kaon decays $K^{+} \rightarrow \pi^{+} v v, K_{L} \rightarrow \pi^{0} v v$, and in the observation of the $\mathrm{C} v \mathrm{~B}$ in the future.

\section{Conclusion}

Neutrino masses are constrained by three experimental observables in $\beta$-decay precision measurements, cosmological observations, and neutrinoless double beta decay searches. The global picture in 2020 is summarized in Fig. 4. Recent updates come from the KATRIN experiment which started its $5 \mathrm{yr}$ measurement campaign and published its first leading result of $m_{\beta}<1100 \mathrm{meV}$ in 2019. Cosmological observations give the strongest constraint with $m_{\Sigma}<120 \mathrm{meV}$, which depends on model assumptions and the choice of datasets. $0 v \beta \beta$ decay experiments mainly test lepton number violation, but they can constrain $m_{\beta \beta}$ under the specific assumption of light Majorana neutrino exchange. Many experiments reported incremental progress with the leading constraints coming from the KamLAND-Zen 400 experiment with $m_{\beta \beta}<61-165 \mathrm{meV}$ in ${ }^{136} \mathrm{Xe}$ and from the GERDA experiment with $T_{1 / 2}>1.8 \cdot 10^{26} \mathrm{yr}$ in ${ }^{76} \mathrm{Ge}$.

\section{References}

[1] C. L. Cowan et al., Science 124, 1 (1956).

[2] F. Reines and C. L. Cowan, Nature 178, 4531, 446 (1956).

[3] E. Majorana, Il Nuovo Cimento (in Italian) 14, 171 (1937).

[4] P.A. Zyla et al. (Particle Data Group), Prog. Theor. Exp. Phys. 2020, 083 C01 (2020).

[5] M. Aker et al., Phys. Ref. Lett. 123, 221802 (2019)/ 

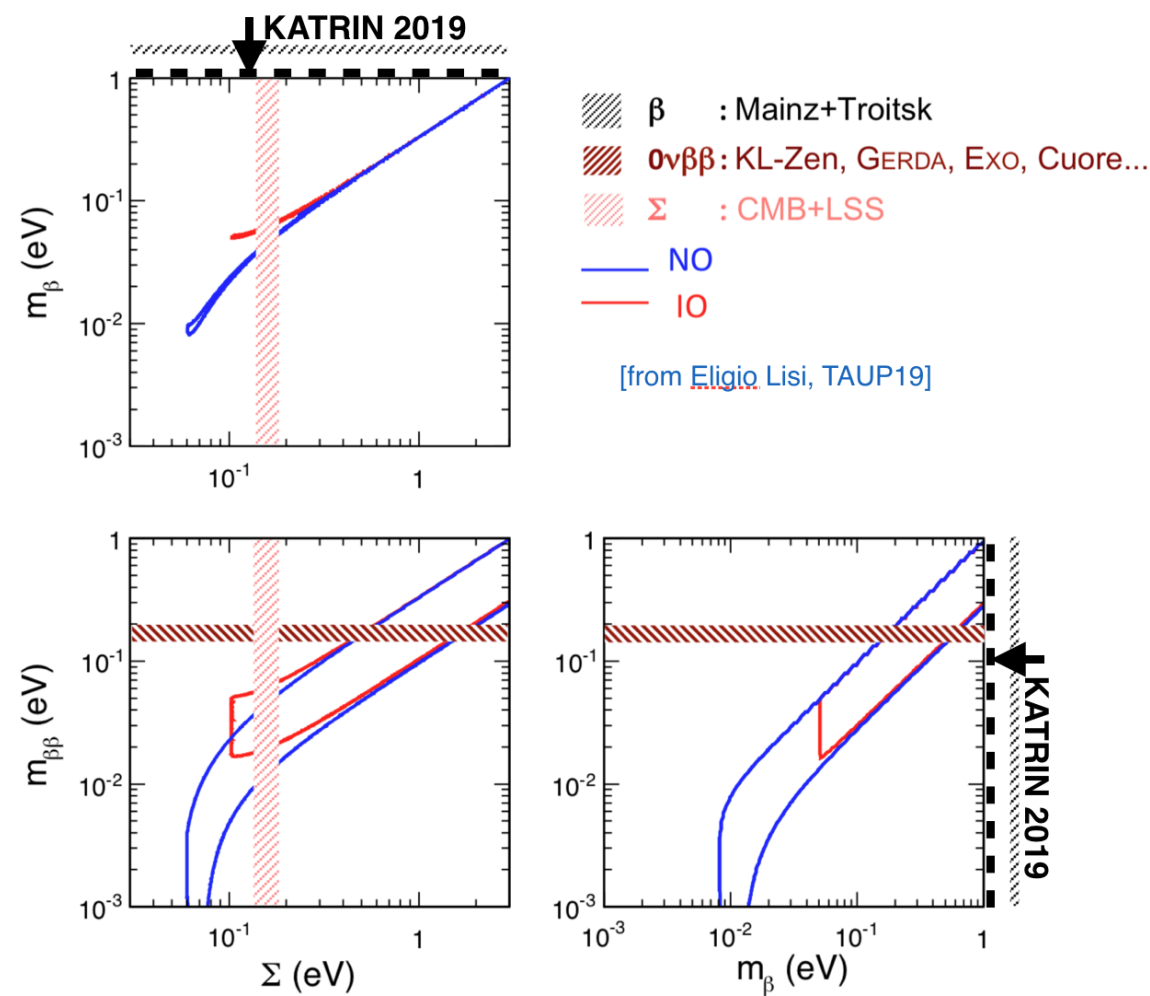

Figure 4: Global picture of parameter spaces for all three neutrino mass observables. Allowed regions from oscillations are shown in blue (NO) and red (IO). Regions with $m_{\beta \beta}$ are wider due to unknown Majorana phases. Regions with $m_{\beta}$ and $m_{\Sigma}$ have lower limits from non-zero mass eigenstates. Cosmological constraints on $m_{\Sigma}$ are the strongest. Kinematic constraints on $m_{\beta}$ are the weakest but the only model independent information in the picture. Adopted from [29].

[6] V. Lokhov and F. V. Tkachov, Phys. Part. Nucl. 46, 347 (2015).

[7] G. J. Feldman and R. D. Cousins, Phys. Rev. D 57, 3873 (1998).

[8] N. Oblath, Neutrino2020, 10.5281/zenodo. 4134024

[9] L. Gastaldo et al., Eur. Phys. J. Spec. Top. 226, 1623 (2017).

[10] A. Orlando et al., J. Low Temp. Phys. 184, 892 (2016).

[11] M. Haverkort, Neutrino2020, 10.5281/zenodo . 4142060

[12] Planck Collaboration, N. Aghanim et al., A\&A, 641 (2020) A6

[13] K. Abazajian et al., arXiv:1907.04473 [astro-ph.IM] (2019).

[14] M.G. Betti et al., J. of Cosm. and Astrop. Phys. 07, 47 (2019).

[15] J. Schechter and J.W.F. Valle, Phys. Rev. D 25, 11 (1982).

[16] M. Duerr, M. Lindner, and A. Merle, J. High Energ. Phys. 6, 91 (2011). 
[17] B. Kayser and G. Segre, Phys. Lett. B 704, 570, (2011).

[18] M. Agostini, G. Benato and J. Detwiler, Phys. Rev. D 96, 053001 (2017).

[19] J. Menéndez, Neutrino2020, 10.5281/zenodo. 4135360

[20] A. Gando et al., Phys. Rev. Lett. 122, 192501 (2019).

[21] B. Lehnert, EPJ WEb of Conf. 93, 01025 (2015).

[22] J. Detwiler, Neutrino2020, 10.5281/zenodo.4154380

[23] C. Grant;, Neutrino2020, 10.5281/zenodo. 4142683

[24] J.J. Gomez-Cadenas, Neutrino2020, 10 . 5281/zenodo. 4142684

[25] XENON Collaboration, Nature 568, 532 (2019).

[26] T. O’Donnell, Neutrino2020, 10.5281/zenodo. 4134025

[27] Y. Kermaidic, Neutrino2020, 10.5281/zenodo.4140757

[28] A. Faessler et al., Phys. Rev. D 83, 113003 (2011).

[29] E. Lisi, TAUP2019, http://www-kam2.icrr.u-tokyo.ac.jp/indico/event/3/ contribution $/ 18$ 